\title{
Oestrogen inhibits PTPRO to prevent the apoptosis of renal podocytes
}

\author{
WEI REN ${ }^{1}$, HUIRU YI ${ }^{1}$, YING BAO ${ }^{2}$, YINGRU LIU ${ }^{1}$ and XINRU GAO ${ }^{3}$ \\ Departments of ${ }^{1}$ Internal Medicine and ${ }^{2}$ Nephrology, Xi'an Children's Hospital, Xi'an, Shaanxi 710003; \\ ${ }^{3}$ Department of Medical Ultrasound Center, The Northwest Women's and \\ Children's Hospital, Xi'an, Shaanxi 710061, P.R. China
}

Received February 11, 2018; Accepted November 7, 2018

DOI: $10.3892 /$ etm.2019.7167

\begin{abstract}
Podocytes are a major component of the glomerular filtration membrane, and their apoptosis is involved in a variety of nephrotic syndromes. In the current study, the effects and molecular mechanisms of oestrogen on the proliferation and apoptosis of podocytes were investigated to elucidate the role of oestrogen in the pathogenesis of childhood nephrotic syndrome. The cell proliferation of mouse renal podocytes (MPC-5) and human primary renal podocytes was promoted by $17 \beta$-oestradiol (E2) in what appear to be a time-dependent manner. Apoptosis was inhibited by E2 and promoted by the E2 antagonist, tamoxifen. The expression of protein tyrosine phosphatase receptor type O (PTPRO) decreased with the increasing dosage of $\mathrm{E} 2$, but increased with the increasing dosage tamoxifen in MPC-5 and human podocytes. The protein, oestrogen receptor (ER) $\alpha$, was not expressed in MPC-5 and human podocytes. E2 binding to ER $\beta$ completely eliminated PTPRO expression in MPC-5. In podocytes, PTPRO was phosphorylated by E2 at the Y1007 and associated with tyrosine-protein kinase JAK2 (JAK2) activation, rather than JAK1 activation. PTPRO was involved in the binding of E2 to signal transducer and activator of transcription (STAT)3 at the Y705 and S727 sites, resulting in the phosphorylation of STAT3 in podocytes. Through PTPRO, E2 also regulated the proliferation and apoptosis of podocytes. In conclusion, oestrogen binding to $\mathrm{ER} \beta$, rather than $\mathrm{ER} \alpha$, promoted the proliferation of podocytes and inhibited the apoptosis of podocytes by inhibiting the expression of PTPRO. The mechanism may be associated with the activation of the JAK2/STAT3 signalling pathway. The current study may provide a novel direction for the treatment of childhood nephrotic syndrome.
\end{abstract}

Correspondence to: Ms. Xinru Gao, Department of Medical Ultrasound Center, The Northwest Women's and Children's Hospital, 1616 Yan Xiang Road, Xi'an, Shaanxi 710061, P.R. China

E-mail: renwei_1014@163.com

Key words: oestrogen, protein tyrosine phosphatase receptor type $\mathrm{O}$, cell proliferation, apoptosis, renal podocytes

\section{Introduction}

Renal podocytes serve a crucial role in glomerular filtration and constitute the major component of the filtration barrier (1). In a study of patients with Alzheimer's disease, podocyte exfoliation from the glomerulus was accelerated with increasing age and the number of podocytes decreased with age (2). The reduction of podocyte number and density induced diseases, including proteinuria, glomerulosclerosis and renal dysfunction (3). Oestrogen affects a variety of physiological and pathological functions of the kidney, including the regulation of haemodynamics, mesangial cells, the mesangial matrix, collagen metabolism, cytokines, the release of inflammatory mediators and glomerular filtration $(4,5)$. Studies have revealed that oestrogen inhibits glomerular podocyte apoptosis through oestrogen-associated receptors $(6,7)$.

The protein tyrosine phosphatase receptor type O (PTPRO) is a type of phosphotyrosine protein phosphatase (PTP) receptor that was first identified and cloned in the human glomerulus (8). PTPRO has six protein subtypes, of which the full-length subtype is expressed in several organs, including the kidneys, brain, lungs, liver and mammary glands, while one of the truncated subtypes is mainly expressed in macrophages and lymphocytes (9). PTPRO is a transmembrane protein, and its intracellular domain contains the PTP domain that catalyses the dephosphorylation of tyrosine residues (8). PTP dephosphorylation is indispensable in cell signal transduction, which greatly influences and regulates the biological behaviour of cells, including cell proliferation, differentiation and apoptosis $(10,11)$. One study demonstrated that the increased expression of PTPRO in oestrogen-induced tumorigenesis could facilitate endocrine therapy in breast cancer (12). Mutations in PTPRO are a cause of autosomal-recessive nephrotic syndrome (13). Additionally, antibodies directed against PTPRO caused increased glomerular protein permeability (14). In particular, antibodies to phosphatases of the extracellular domain resulted in impairment of the permeability barrier (14). These studies indicate that oestrogen mediates glomerular dysfunction, which may be associated with the regulation of PTPRO, however the mechanism remains unclear. The current study mainly investigated the effect of oestrogen on the apoptosis of renal podocytes, but also explored its possible signal transduction mechanism, which may provide a novel target for the treatment of childhood nephrotic syndrome. 


\section{Materials and methods}

Cell culture and treatment. A mouse podocyte cell line derived from kidneys (MPC5) was obtained from the Biotechnology Co., Ltd. Shanghai Enzyme Research (Shanghai, China). Human primary renal podocytes derived from kidneys (HUM-iCELL-u004) were obtained from iCell Bioscience Inc. (Shanghai, China). All cells were cultured in Dulbecco's modified Eagle's medium (DMEM) supplemented with $10 \%$ foetal bovine serum (FBS), $100 \mathrm{U} / \mathrm{ml}$ penicillin and $100 \mathrm{mg} / \mathrm{ml}$ streptomycin (all Invitrogen; Thermo Fisher Scientific, Inc., Waltham, MA, USA), and maintained at $37^{\circ} \mathrm{C}$ in a humidified incubator with $5 \% \mathrm{CO}_{2}$.

Cells were washed once with PBS prior to stimulation with $17 \beta$-oestradiol (E2) at dosages of $5,10,50$ or $100 \mathrm{nM}$, or the E2 antagonist, tamoxifen, (both Sigma-Aldrich; Merck KGaA, Darmstadt, Germany) at dosages of 0.1 or $5 \mu \mathrm{M}$ for 7 days at $37^{\circ} \mathrm{C}$. During stimulation, cell viability and proliferation were analysed by the MTT assay, and apoptosis were analysed by flow cytometry.

Construction of the PTPRO overexpression vector. Cells were transfected with PTPRO or ER $\beta$ overexpression vectors 5 days after the stimulation. The pcDNA3.1(+)/PTPRO expression vector was constructed by cloning a PTPRO fragment from normal mouse cDNA (Sangon Biotech Co., Ltd., Shanghai, China) into pcDNA3.1(+; Invitrogen; Thermo Fisher Scientific, Inc.) between the BamH I and EcoR I sites to express PTPRO in abundance in E. coli DH5 $\alpha$ cells (Takara Biotechnology Co., Ltd., Dalian, China). The primers for PTPRO were as follows: Forward: 5'-GGAACCACTGACCTGTCCCACTC-3', reverse: 5'-CTCGGTGTTGCTCCCTCTCTCAG-3'. Then, the $1 \mu \mathrm{g}$ pcDNA3.1(+)/PTPRO plasmid was transfected into MPC5 cells using Lipofectamine 2000 (Invitrogen; Thermo Fisher Scientific, Inc.). The stably transfected clones were screened for G418 resistance using $50 \mathrm{mg} / \mathrm{ml}$ Geneticin ${ }^{\mathrm{TM}}$ Selective Antibiotic (G418 Sulfate; Invitrogen; Thermo Fisher Scientific, Inc.) at $24 \mathrm{~h}$ after transfection. Briefly, cells were cultured after $24 \mathrm{~h}$ of transfection in DMEM with $1,200 \mu \mathrm{g} / \mathrm{ml}$ $\mathrm{G} 418$ sulfate at $37^{\circ} \mathrm{C}$ for 14 days, and then the positive clones were observed with a light microscope at a magnification of $x 200$. The target gene was then detected by western blot analysis. The pcDNA3.1(+)/ER $\beta$ expression vector was constructed by cloning a ER $\beta$ fragment from normal mouse cDNA (Sangon Biotech Co., Ltd.) into the pcDNA3.1(+) plasmid (Invitrogen; Thermo Fisher Scientific, Inc.). Then $1 \mu \mathrm{g}$ pcDNA3.1(+)/ER $\beta$ plasmid or pcDNA3.1(+) plasmid (control) were transfected into MPC5 cells with or without E2 treatment using Lipofectamine 2000 at $37^{\circ} \mathrm{C}$ for $24 \mathrm{~h}$. The primers for ER $\beta$ were as follows: Forward: 5'-CCTCGTTCTGGACAG GGATG-3', reverse: 5'-AGAAGCATCAGGAGGTTGGC-3'.

Semi-quantitative reverse transcription-polymerase chain reaction ( $R T-P C R)$. MPC-5 cells or human primary renal podocytes were treated with $5,10,50$ or $100 \mathrm{nM} \mathrm{E} 2$ or 0.1 or $5 \mu \mathrm{M}$ tamoxifen. Total RNA was extracted from cells using TRIzol reagent (Invitrogen; Thermo Fisher Scientific, Inc.). Isolated RNA was electrophoresed in a $1 \%$ agarose gel to detect the purity of total RNA. The first-strand cDNA was synthesized in a $10 \mu \mathrm{l}$ reaction system using $1 \mu \mathrm{g}$ total RNA and SuperScript ${ }^{\circledR}$ III Reverse Transcriptase (Invitrogen; Thermo Fisher Scientific, Inc.), and placed in a PCR thermocycler at $37^{\circ} \mathrm{C}$ for $60 \mathrm{~min}$. PCR amplification was performed using the PCR amplification kit (Taq; Takara Biotechnology Co., Ltd., Dalian, China). The thermocycling conditions were as follows: $94^{\circ} \mathrm{C}$ for $5 \mathrm{~min}$, then 30 cycles of $94^{\circ} \mathrm{C}$ for $45 \mathrm{sec}$, $56^{\circ} \mathrm{C}$ for $45 \mathrm{sec}$ and $72^{\circ} \mathrm{C}$ for $45 \mathrm{sec}$. The number of cycles used was determined by comparing the results of RT-PCR analyses with different numbers of cycles (Fig. 1). With the gradual accumulation of PCR products, the amplified DNA fragments no longer increase exponentially, and enter the linear growth phase or stationary phase, that is the 'stagnation effect', it named as plateau phase (15). When cells were in the plateau phase, the relative mRNA expression identified in the 30 -cycle analysis was similar to that of the 35 -cycle analysis. However, the relative mRNA expression identified in the 30 -cycle analysis was significantly higher compared with that of 28-cycle analysis and significantly lower compared with that of 35-cycle analysis (both $\mathrm{P}<0.05$ ). Therefore, 30 cycles were in the exponential phase. The specific primers were designed using Primer Premier 6.0 software (Premier Biosoft International, Inc., Palo Alto, CA, USA) and synthesized by Sangon Biotech Co., Ltd. The primers for PTPRO (mouse) were as follows: Forward: 5'-ACCACTGACCTGTCCCAC TC-3'; reverse: 5'-AGGTGTTGCTCCCTCTCTCA-3'. The primers for PTPRO (human) were as follows: Forward: 5'-TCTGCAGATGGCTAGGGAGT-3'; reverse: 5'-AGACAT GAGGGTAGCAGGGT-3'. The primers for $\beta$-actin (human) were as follows: Forward: 5'-CCGTTCCGAAAGTTGCCT TTT-3'; reverse: 5'-GAGGCGTACAGGGATAGCAC-3'. The PCR product was electrophoresed in a $1 \%$ agarose gel and the bands were visualized by ethidium-bromide staining. Bio-Rad Gel Imaging System (Bio-Rad Laboratories, Inc., Hercules, CA, USA) was used to observe the bands. Each band was analysed by Quantity One 4.62 software (Bio-Rad Laboratories, Inc.). The intensity of the target band were normalised to the $\beta$-actin band.

Western blotting. MPC- 5 cells were treated by $100 \mathrm{nM}$ E2 or PBS (control) for 5 days, and then were incubated with pcDNA3.1(+)/PTPRO expression vector or pcDNA3.1(+) scramble plasmids. Subsequently, total proteins were extracted from cells using Cell Total Protein Extraction kit (Amresco, LLC, Solon, OH, USA) and quantified with Bicinchoninic Acid Protein Concentration Determination kit (Beyotime Institute of Biotechnology, Shanghai, China). All antibodies were purchased from Abcam (Cambridge, UK). The proteins ( $20 \mu \mathrm{g} /$ lane) were separated by SDS-PAGE in a $10 \%$ gel followed by electrotransfer to nitrocellulose membranes. The membranes were blocked by $5 \% \mathrm{FBS}$ at room temperature for $45 \mathrm{~min}$, and probed using primary antibodies against PTPRO (1:500; cat. no. ab231560), ER $\beta$ (1:1,000; cat. no. ab3577), signal transducer and activator of transcription (STAT3; 1:1,000; cat. no. ab68153), phosphorylated (p-)STAT3 (Y705; 1:1,000; cat. no. ab76315), p-STAT3 (S727; 1:2,000; cat. no. ab30647), tyrosine-protein kinase JAK1 (JAK1; 1:1,000; cat. no. ab47435), p-JAK1 (Y1022+Y1023; 1:1,000; cat. no. ab138005), JAK2 (1:5,000; cat. no. ab39636), p-JAK2 (Y1007; 1:1,000; cat. no. ab195055) and $\beta$-actin $(1: 10,000$; cat. no. ab8227) overnight at $4^{\circ} \mathrm{C}$. They were also probed using 


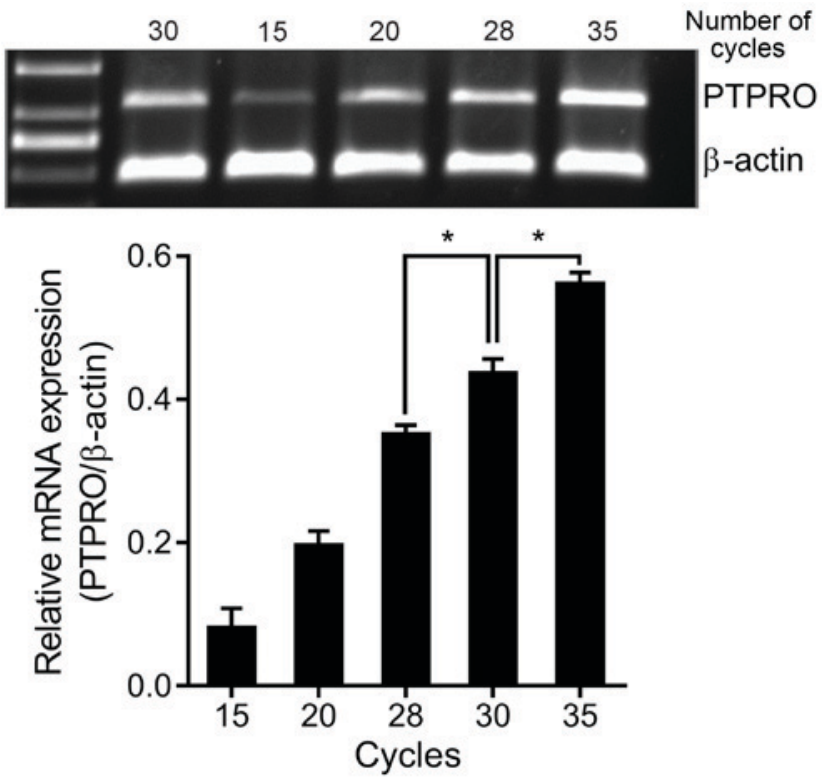

Figure 1. Identification of the number of cycles for PCR analysis. The number of cycles used for the PCR was determined using semi-quantitative RT-PCR. ${ }^{*} \mathrm{P}<0.05$ as indicated. RT-PCR, reverse transcription-polymerase chain reaction.

primary antibodies against $\operatorname{ER} \alpha(1: 600$; cat. no. ab75635) for $4 \mathrm{~h}$ at room temperature. The membranes were then incubated horseradish peroxidase-conjugated secondary antibodies (1:20,000; cat. no. ab7090) at room temperature for $1 \mathrm{~h}$. $\beta$-actin was used as an internal reference. Bands were revealed with an Electro-Chemi-Luminescence (ECL) reagent (EMD Millipore, Billerica, MA, USA) and recorded on X-ray films (Kodak, Rochester, NY, USA). The densitometry of each band was quantified using a Gel imaging system and Quantity One 4.62 software.

Cell viability and proliferation assays. After cells were incubated with E2 or tamoxifen for 5 days, cell viability and proliferation was analysed using MTT Cell Proliferation and Cytotoxicity Assay kit (Beyotime Institute of Biotechnology) every day for 7 days. The dimethyl sulfoxide was used to dissolve the purple formazan. The optical density value was recorded at a wavelength of $450 \mathrm{~nm}$. Then, time was plotted on the abscissa and absorbance on the ordinate to plot a cell growth curve. The assay was repeated four times for each sample.

Cell apoptosis assay. After cells were incubated with E2 or tamoxifen for 5 days, apoptotic cells were measured by flow cytometry using an Annexin V-fluorescein isothiocyanate/propidium iodide apoptosis detection kit (Abcam). The fluorescence intensity was detected at $488 \mathrm{~nm}$ using flow cytometry. Cells were sorted by the FACSCalibur flow cytometer and analysed using CellQuest software (version 5.1; both BD Biosciences, Franklin Lakes, NJ, USA).

Statistical analysis. Data are presented as mean \pm standard deviation of at least four replicates per group. One-way analysis of variance and Fisher's Least Significant Difference were used to compare multiple groups. Data were analysed by
SPSS 13.0 software (SPSS, Inc., Chicago, IL, USA). Statistical differences were calculated using t-tests in Fig. 3D-G.

\section{Results}

Oestrogen promotes cell viability and proliferation, and prevents apoptosis in podocytes. Cell viability increased with increasing E2 dosages, but decreased with increasing dosages of tamoxifen in MPC-5 cells (Fig. 2A); these changes were significant compared with the control cells. After 4 days, cell proliferation significantly increased with the duration of $100 \mathrm{nM}$ E2 stimulation, whereas, after 3 days, it significantly decreased with the duration of $5 \mu \mathrm{M}$ tamoxifen stimulation in MPC-5 cells compared with control cells (Fig. 2B). E2 (100 nM) stimulation significantly inhibited apoptosis, whereas tamoxifen $(5 \mu \mathrm{M})$ stimulation significantly accelerated apoptosis in MPC-5 cells (Fig. 2C). Cell proliferation increased with increasing E2 dosages, but decreased with increasing dosages of tamoxifen in human primary renal podocytes (Fig. 2D); these changes were significant compared with the control cells. After 5 days, cell proliferation significantly increased with the duration of $100 \mathrm{nM}$ E2 stimulation, whereas, after 4 days, it significantly decreased with the duration of $5 \mu \mathrm{M}$ tamoxifen stimulation in MPC-5 cells compared with control cells (Fig. 2B). E2 (100 nM) stimulation significantly inhibited apoptosis, whereas tamoxifen $(5 \mu \mathrm{M})$ stimulation significantly accelerated apoptosis human primary renal podocytes (Fig. 2F). These results indicate that $\mathrm{E} 2$ can promote cell viability and proliferation, and prevent the apoptosis of podocytes. Additionally, it was also determined that the effect of oestrogen on viability, proliferation and apoptosis were the same in mouse cell lines (MPC-5) as in human cell lines (human primary renal podocytes), suggesting that the effect was not species-specific.

Oestrogen and its receptors inhibit PTPRO expression in podocytes. The mRNA expression levels of PTPRO decreased with increasing E2 dosages, but this was only significant with $100 \mathrm{nM}$ E2 in MPC-5 cells, and 50 and $100 \mathrm{nM} \mathrm{E} 2$ in human primary renal podocytes compared with the controls (Fig. 3A and B). PTPRO mRNA expression increased with $5 \mu \mathrm{M}$ tamoxifen in MPC-5 cells, and with 0.1 and $5 \mu \mathrm{M}$ tamoxifen in human primary renal podocytes compared with the controls. These results indicate that E2 can inhibit the expression of PTPRO in podocytes.

To explore the role of oestrogen receptors in regulating PTPRO expression, the expression levels of ER $\alpha$ and ER $\beta$ proteins were detected in MPC-5 cells and human primary renal podocytes. The results demonstrated that the ER $\alpha$ protein was not expressed, while the ER $\beta$ protein was expressed in MPC-5 cells and human primary renal podocytes (Fig. 3C and D). Subsequently, the ER $\beta$ overexpression vectors were transfected into E2 (100 nM)-stimulated and non-stimulated MPC5 cells, and the transfection effect was detected by western blot analysis (Fig. 3C and E). Compared with control cells, ER $\beta$ expression was significantly increased by ER $\beta$ overexpression vectors in E2-stimulated and non-stimulated MPC-5 cells. The binding of E2 to increased levels of ER $\beta$ significantly eliminated PTPRO mRNA and protein expression compared with 

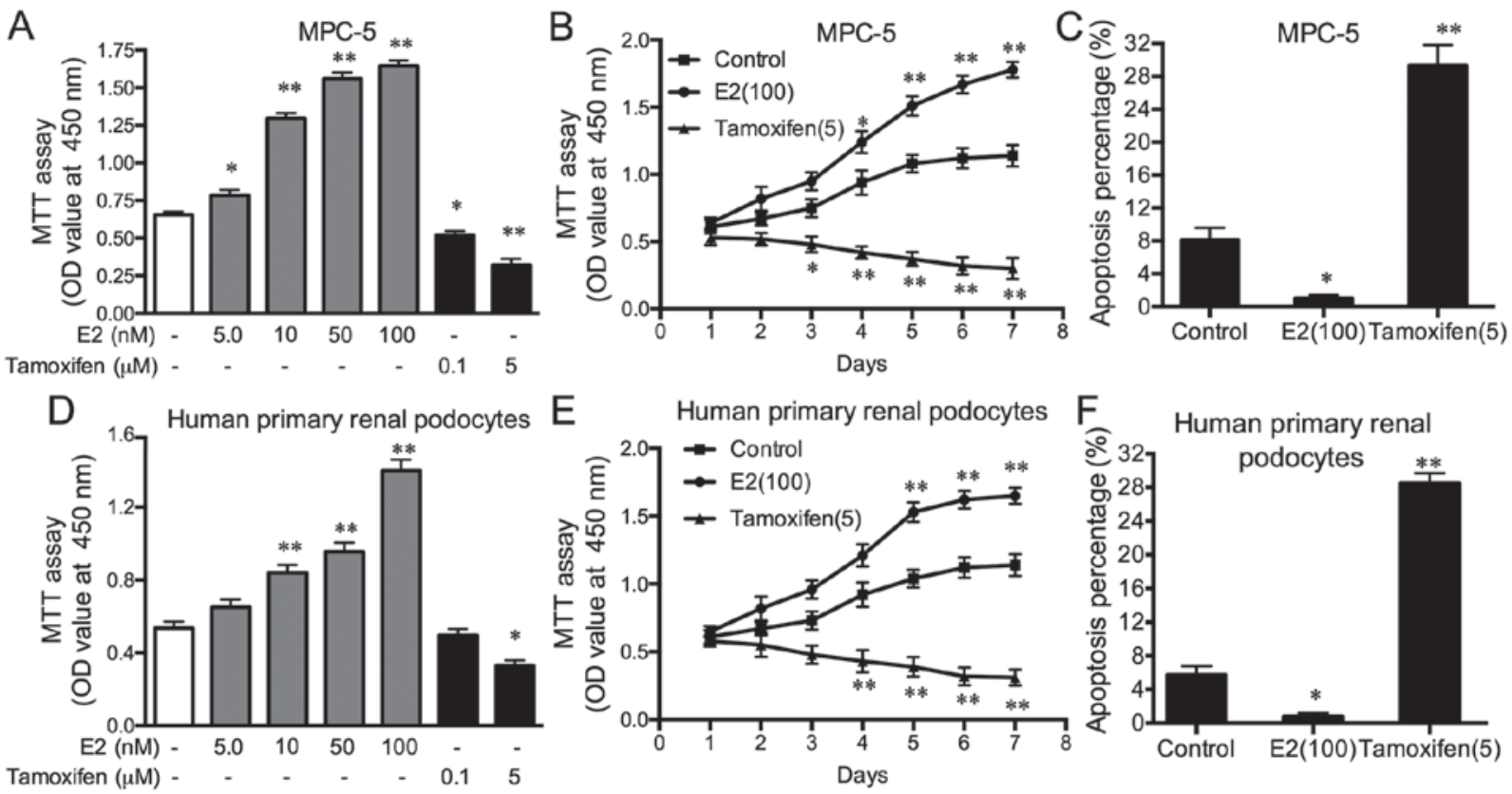

Figure 2. Oestrogen promotes cell viability and proliferation, and prevents apoptosis in podocytes. (A) The viability of MPC-5 cells treated with 5-100 nM E2 or 0.1-5 $\mu \mathrm{M}$ tamoxifen using a MTT assay. The (B) proliferation rate of and (C) percentage of apoptotic MPC-5 cells treated with $100 \mathrm{nM}$ E2 or $5 \mu \mathrm{M}$ tamoxifen using a MTT assay and flow cytometry, respectively. (D) The viability of human primary renal podocytes treated with 5-100 nM E2 or $0.1-5 \mu \mathrm{M}$ tamoxifen using a MTT assay. The (E) proliferation rate of and (F) percentage of apoptotic human primary renal podocytes treated with $100 \mathrm{nM}$ E2 or $5 \mu \mathrm{M}$ tamoxifen using a MTT assay and flow cytometry, respectively. ${ }^{*} \mathrm{P}<0.05,{ }^{* *} \mathrm{P}<0.01$ vs. Control. E2, 17 $\beta$-oestradiol.

A

A $\quad$ B
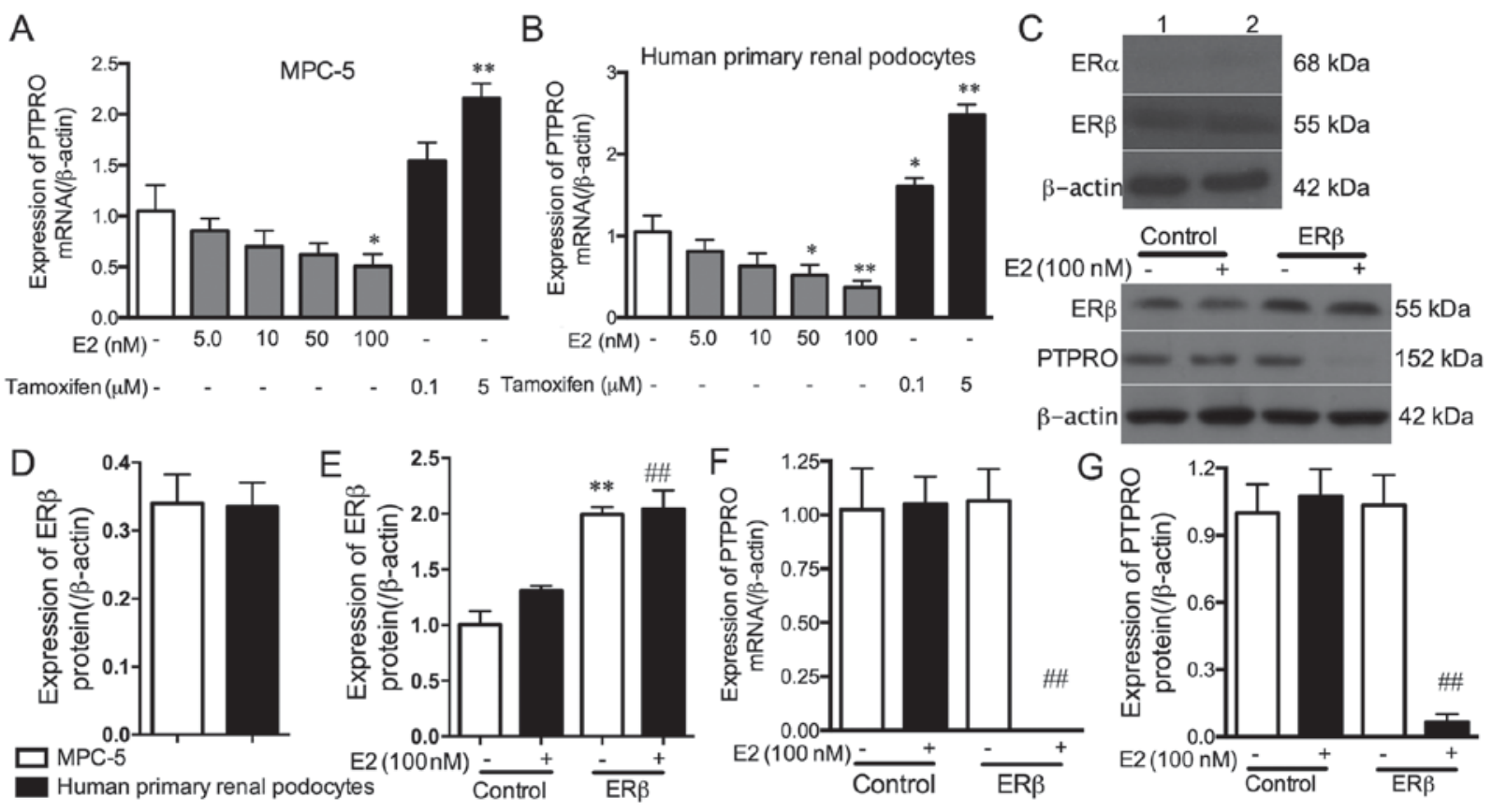

Figure 3. Oestrogen and its receptors inhibit PTPRO expression in podocytes. PTPRO mRNA expression in (A) MPC-5 cells and (B) human primary renal podocytes treated with $5-100 \mathrm{nM} \mathrm{E} 2$ or $0.1-5 \mu \mathrm{M}$ tamoxifen using semi-quantitative reverse transcription-polymerase chain reaction. ${ }^{*} \mathrm{P}<0.05$, ${ }^{* *} \mathrm{P}<0.01$ vs. Control. (C) The protein expression of ER $\alpha$ and ER $\beta$ were detected by western blotting in MPC-5 cells and human primary renal podocytes. The protein expression of ER $\beta$ and PTPRO were detected by western blotting in MPC-5 cells stimulated with $100 \mathrm{nM}$ E2 and/or ER $\beta$ overexpression vectors. (D) The quantification of ER $\alpha$ and ER $\beta$ in MPC-5 cells and human primary renal podocytes. (E) The quantification of the ER $\beta$ protein in MPC-5 cells stimulated with $100 \mathrm{nM}$ E2 and/or ER $\beta$ overexpression vectors. The quantification of (F) the PTPRO mRNA and (G) the PTPRO protein in MPC-5 cells stimulated with $100 \mathrm{nM}$ E2 and/or an ER $\beta$ overexpression vector. ${ }^{* *} \mathrm{P}<0.01$ vs. Control-; ${ }^{\# \#} \mathrm{P}<0.01$ vs. Control+. PTPRO, protein tyrosine phosphatase receptor type O; E2, 17 $\beta$-oestradiol; ER, oestrogen receptor; 1, MPC-5 cells; 2 , human primary renal podocytes.

E2 (100 nM)-stimulated cells (Fig. 3C, F and G). These results suggest that E2 combines with ER $\beta$ rather than ER $\alpha$, to inhibit PTPRO expression in podocytes.
Oestrogen activates JAK2 by inhibiting PTPRO in mouse podocytes. To further explore the molecular mechanism by which E2 regulates PTPRO expression in podocytes, protein 

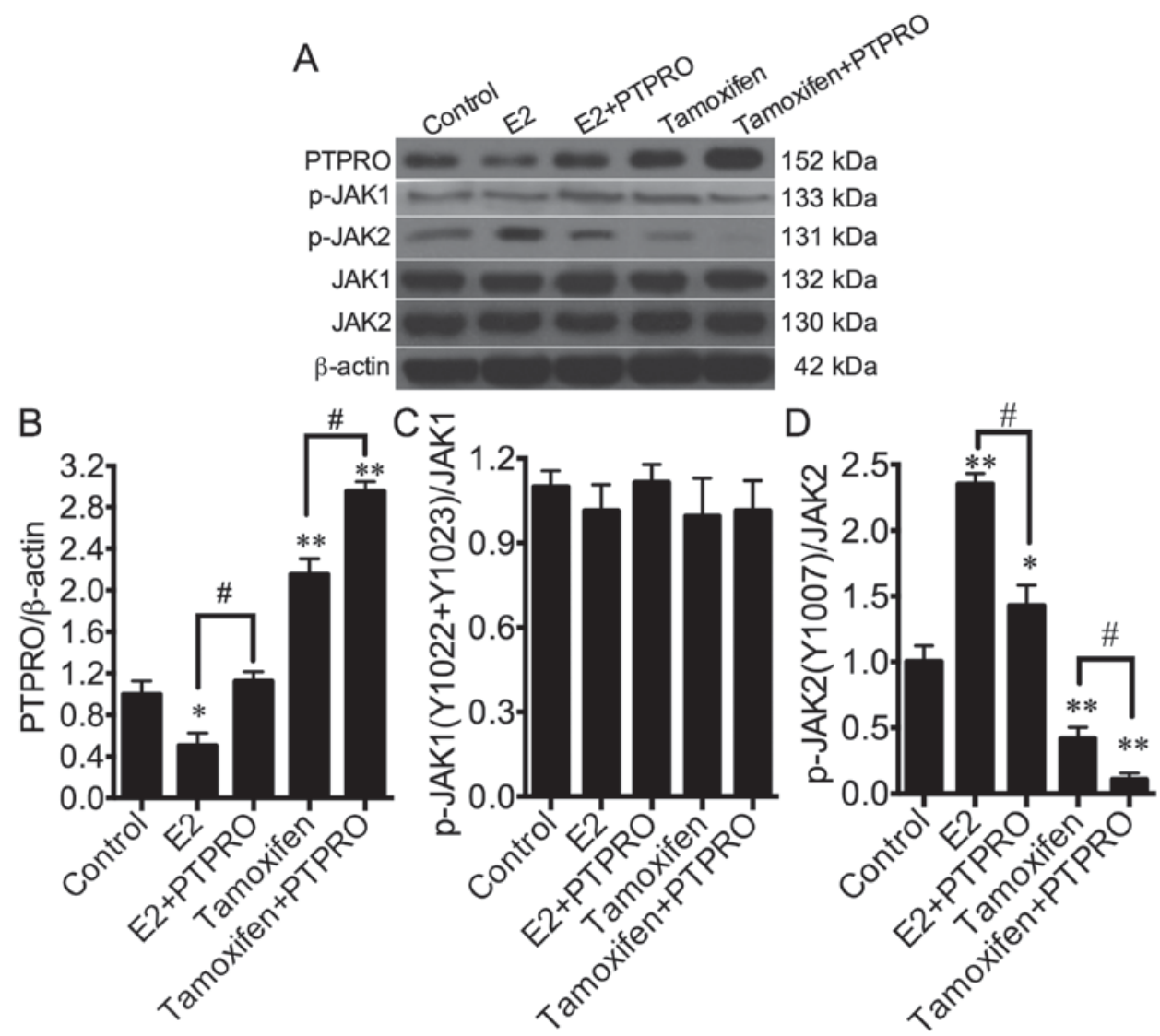

Figure 4. Oestrogen activates JAK2 by inhibiting PTPRO in podocytes. MPC-5 cells were simulated with in E2 or tamoxifen and transfected with a PTPRO overexpression vector. (A) Protein expression in MPC5 cells. Quantification of (B) PTPRO, (C) p-JAK1 and (D) p-JAK2 proteins in MPC5 cells. "P<0.05, ${ }^{* *} \mathrm{P}<0.01$ vs. Control; ${ }^{*} \mathrm{P}<0.05$ as indicated. PTPRO, protein tyrosine phosphatase receptor type O; E2, 17ß-oestradiol; ER, oestrogen receptor; p-, phosphorylation; JAK, tyrosine-protein kinase JAK.

expression levels of PTPRO, p-JAK1 and p-JAK2 were analysed. Firstly, PTPRO overexpression vectors were transfected into E2-stimulated or tamoxifen-stimulated MPC-5 cells, then PTPRO expression was detected by western blot analysis. PTPRO overexpression vectors significantly promoted E2-inhibited and tamoxifen-inhibited PTPRO expression (Fig. 4A and B). Phosphorylation of the Y1022 and Y1023 sites of JAK1 was not affected by E2 or tamoxifen stimulation with or without PTPRO overexpression (Fig. 4A and C). E2 significantly increased the expression of JAK2 phosphorylated at the Y1007 compared with the control, while PTPRO overexpression significantly dephosphorylated the Y1007 site of JAK2 in E2-stimulated MPC-5 cells (Fig. 4A and D). Additionally, tamoxifen significantly dephosphorylated the Y1007 site of JAK2 compared with the control. PTPRO overexpression significantly dephosphorylated the Y1007 site of JAK2 in tamoxifen-stimulated MPC-5 cells. These results suggest that PTPRO is involved in E2-induced JAK2 activation in podocytes, rather than JAK1 activation.

Oestrogen activates STAT3 by inhibiting PTPRO in podocytes. Previous studies have revealed that the suppressive role of PTPRO in hepatocellular carcinoma or breast cancer could be ascribed to the regulation of STAT3 expression (16). Therefore, in the current study, to elucidate the underlying mechanism by which PTPRO is involved in the inhibition of podocyte viability by oestrogen, the regulation of STAT3 activity by PTPRO was analysed.

E2 significantly phosphorylated the Y705 and S727 sites of STAT3 compared with the control, and PTPRO overexpression significantly dephosphorylated the Y705 and S727 sites of STAT3 in E2-stimulated MPC-5 cells (Fig. 5A-C). p-STAT3(Y705) expression was significantly increased in E2-stimulated and PTPRO overexpressed MPC-5 cells compared with the control, whereas p-STAT3(S727) expression was only markedly increased. Additionally, tamoxifen significantly dephosphorylated Y705 and S727 sites of STAT3 compared with the control, and PTPRO overexpression significantly dephosphorylated the Y705 and S727 sites of STAT3 in tamoxifen-stimulated MPC-5 cells. These results indicate that PTPRO is involved in E2-induced STAT3 phosphorylation.

PTPRO is involved in the regulation of the viability and apoptosis of podocytes through E2 binding. E2 significantly promoted cell viability and prevented apoptosis compared with the controls, while PTPRO overexpression significantly reversed these E2-induced effects (Fig. 5D and E). The E2 antagonist, tamoxifen, significantly suppressed cell viability and promoted apoptosis compared with the control, while PTPRO overexpression significantly amplified these E2-induced effects. These results indicate that PTPRO is involved involved in the regulation of podocyte viability and apoptosis through E2. 

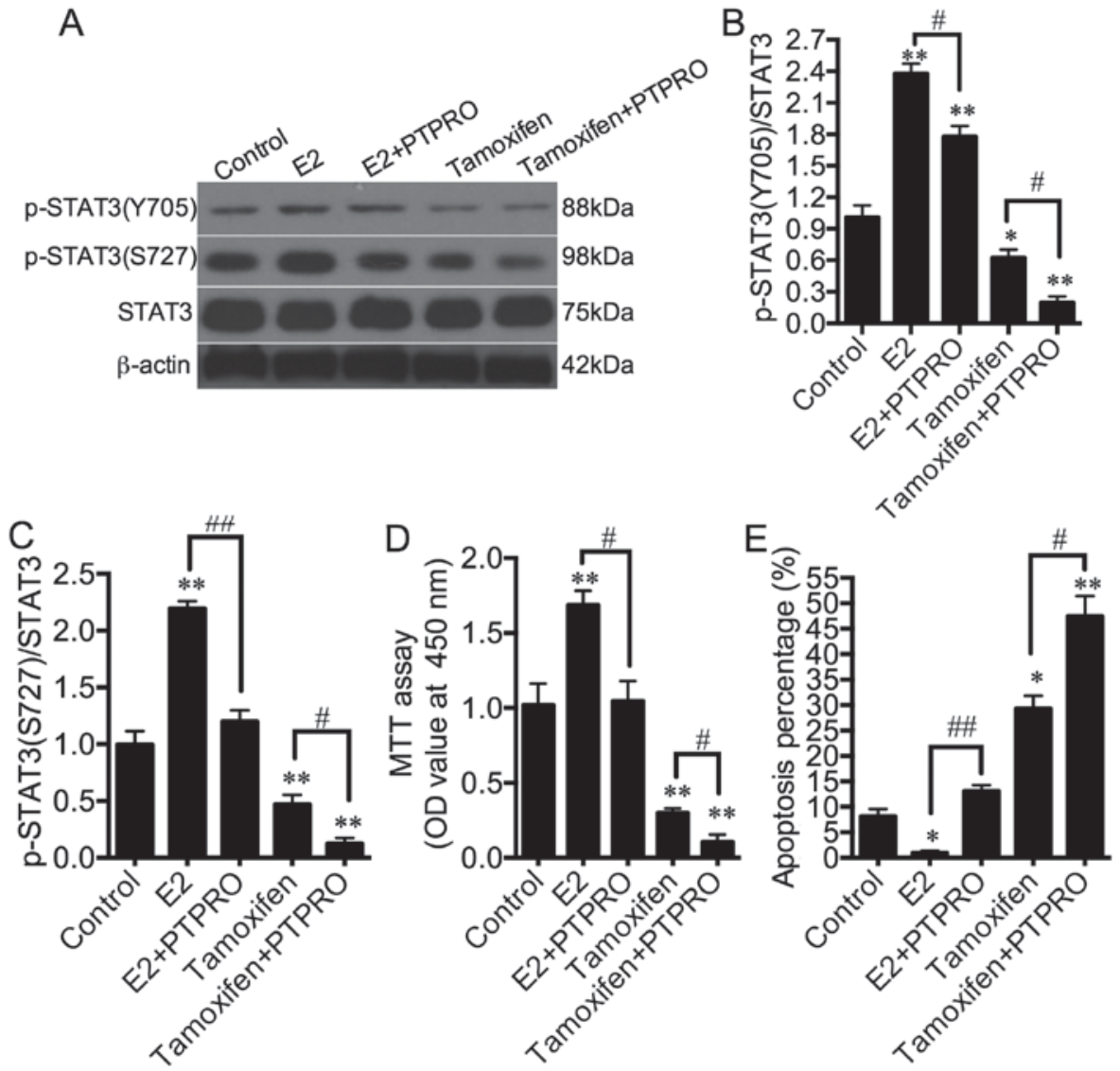

Figure 5. Oestrogen activates STAT3 by inhibiting PTPRO, which is involved in the regulation of the viability and apoptosis of podocytes through E2 binding. MPC-5 cells were simulated with in E2 or tamoxifen and transfected with a PTPRO overexpression vector. (A) Protein expression in MPC5 cells. Quantification of (B) p-STAT3(Y705) and (C) p-STAT3(S727) proteins. The (D) viability of and (E) percentage of apoptotic MPC-5 cells. "P<0.05, ${ }^{* *} \mathrm{P}<0.01$ vs. Control; " $\mathrm{P}<0.05,{ }^{\# \#} \mathrm{P}<0.01$. PTPRO, protein tyrosine phosphatase receptor type O; E2, 17ß-oestradiol; p-, phosphorylation; OD, optical density; STAT, signal transducer and activator of transcription.

\section{Discussion}

Childhood nephrotic syndrome has multiple aetiologies, which induce an increase of glomerular basement membrane permeability and thus a large amount of protein is lost by excretion into the urine $(17,18)$. Podocytes attach to the outer side of the glomerular basement membrane to form the glomerular hemofiltration barrier between vascular endothelial cells and the glomerular basement membrane (19-21). Therefore, podocyte apoptosis will increase glomerular basement membrane permeability, inducing proteinuria $(20,22,23)$, which is one of the risk factors for the pathogenesis of childhood nephrotic syndrome. However, little is known about the mechanism of podocyte apoptosis.

In the current study, it was demonstrated that oestrogen promotes podocyte proliferation and inhibits podocyte apoptosis, which are associated with the binding of oestrogen to its receptor, ER $\beta$, rather than ER $\alpha$, to eliminate PTPRO expression. The mechanism may be associated with the activation of the JAK2/STAT3 signalling pathway by oestrogen.

Oestrogen can inhibit or attenuate the progression of chronic kidney disease caused by multiple issues, such as urinary tract obstruction $(24,25)$. A study using a kidney-wrapped hypertension model in rats revealed that the castration of male rats reduced proteinuria, and glomerular and tubular damage, whereas the addition of dihydrotestosterone inhibited this protective effect (26). The sterilization of female rats increased glomerular and tubular damage, the addition of E2 reduced castration-induced kidney damage, and the addition of dihydrotestosterone inhibited the protective effect of E2 (26). Therefore, androgens were demonstrated to aggravate renal injury caused by kidney-wrap-induced hypertension, while oestrogen had a protective effect on the condition. The current study indicated that oestrogen promoted the proliferation of podocytes and inhibited the apoptosis of podocytes.

PTPRO expression has been determined to be highest in the kidneys and brain, but was also high in other tissues, including the liver and mammary glands (27). Studies have revealed that the oestrogen-oestrogen receptor complex (E2-ER) can regulate the transcription of PTPRO, and ER $\alpha$ and ER $\beta$ serve different functions in the regulatory process $(12,16)$. In the current study, although ER $\alpha$ promoted the transcriptional activation of PTPRO, the transcriptional repression of PTPRO by ER $\beta$ was demonstrated to be the more pronounced process. Additionally, ER $\alpha$ was not expressed in MPC-5 and human primary renal podocytes. E2 bound to ER $\beta$ to further induce the separation of c-jun and c-fos from the activator protein 1 site in the promoter region of PTPRO, thereby inhibiting the transcription of PTPRO (12). The aforementioned findings are consistent with the current study, which demonstrated that the expression of PTPRO was decreased with the increase of E2 
dosages in MPC-5 and human primary renal podocytes. In essence, E2 inhibited the expression of PTPRO by binding to $\mathrm{ER} \beta$, rather than $\mathrm{ER} \alpha$.

Studies have revealed that PTPRO downregulation prevents paediatric nephrotic syndrome. Notably, Ozaltin et al (13) reported that mutations in the PTPRO gene caused diffuse podocyte effacement and the extensive microvillus transformation of podocytes, which contributed to the occurrence of childhood nephrotic syndrome. However, another study reported that, although $\mathrm{PTPRO}^{-/}$mice have shortened podocytes and a reduced total slit diaphragm length, they did not appear to develop major proteinuria or present with a reduction of glomerular filtration rate (28). The results of immunofluorescent and western blot analyses revealed that $\mathrm{PTPRO}^{-/}$increased the fluorescence intensity and expression of vimentin in glomeruli (28). Vimentin is a major component of intermediate filaments, which are present in the major processes of podocytes (29). Therefore, these results appeared to suggest that the inhibition of PTPRO promoted the flexibility of podocytes and prevented nephrotic syndrome. The authors of the current study hypothesise that these contradictory results may be associated with organism-specific differences in functional redundancy of some phosphatases.

The activation of STAT3 by tyrosine phosphorylation serves an essential role in the overall process of intracellular signal transduction (30). Studies demonstrated that, when cells undergo sustained stimulation from a variety of cytokines and growth factors, including IL-6 and EGF, their homologous receptors are recruited and activate JAK2 in a tyrosine-phosphorylation-dependent manner, which may also lead to the activation of STAT3 $(31,32)$. Additionally, another well-known tyrosine kinase, c-Src, was revealed to be activated and contributed to STAT3 activation by phosphorylating S727 and T705 in STAT3 $(33,34)$. Some molecular agents or proteins that attenuate STAT3 activity or block upstream phosphorylation cascades may suppress cell growth, such as that of PTPs $(35,36)$. Other proteins or polypeptides, including TROP2 and P4HB, exhibit the opposite effect, contributing to the activation of STAT3 and its upstream signal molecules $(11,12)$. The current study demonstrated that oestrogen activates Y705 and S727 phosphorylation sites of STAT3 and the Y1007 phosphorylation site of JAK2, indicating that the JAK2/STAT3 signalling pathway is involved in oestrogen regulation.

In the current study, oestrogen bound with $\operatorname{ER} \beta$, rather than ER $\alpha$, promoted podocyte proliferation and inhibited podocyte apoptosis by inhibiting the expression of PTPRO and activating the JAK2/STAT3 signalling pathway. The authors suggest that the current study may provide novel ideas for the prevention of childhood nephrotic syndrome. Additionally, it was also determined that PTPRO can activate c-Src, p38 mitogen-activated protein kinase (MAPK) and MAPK signals during the experiment, inferring that these signals may be involved in the regulation of oestrogen in podocyte proliferation (data not shown). Therefore, this inference will be the focus of a future study by our group.

\section{Acknowledgements}

Not applicable.

\section{Funding}

No funding was received.

\section{Availability of data and materials}

The datasets used and/or analysed during the current study are available from the corresponding author on reasonable request.

\section{Author's contributions}

XG and WR conceived of the study. WR, HY, YB and YL performed the experiments, and collected and analysed all data. WR and HY prepared the manuscript, while XG, YB and YL revised the manuscript. All authors edited the manuscript, contributed to the writing of the manuscript, and read and approved the final manuscript.

\section{Ethics approval and consent to participate}

Not applicable.

\section{Patient consent for publication}

Not applicable.

\section{Competing interests}

The authors declare that they have no competing interests.

\section{References}

1. Welsh GI and Saleem MA: Nephrin-signature molecule of the glomerular podocyte? J Pathol 220: 328-237, 2010.

2. Niranjan T, Bielesz B, Gruenwald A, Ponda M P, Kopp JB Thomas DB and Susztak K: The Notch pathway in podocytes plays a role in the development of glomerular disease. Nat Med 14: 290-298, 2008.

3. Ding F, Wickman L, Wang SQ, Zhang Y, Wang F, Afshinnia F, Hodgin J, Ding J, and Wiggins RC: Accelerated podocyte detachment and progressive podocyte loss from glomeruli with age in Alport Syndrome. Kidney Int 92: 1515-1525, 2017.

4. Mauvais-Jarvis F, Clegg DJ and Hevener AL: The role of estrogens in control of energy balance and glucose homeostasis. Endocr Rev 34: 309-338, 2013.

5. Mercantepe T, Unal D, Selli J, Mercantepe F, Unal B and Karabiyik TN: Protective effects of estrogen and bortezomib in kidney tissue of post-menopausal rats: An ultrastructural study. Ren Fail 38: 1129-1135, 2016.

6. Gong W, Yu J, Wang Q, Li S, Song J, Jia Z, Huang S and Zhang A: Estrogen-related receptor (ERR) gamma protects against puromycin aminonucleoside-induced podocyte apoptosis by targeting PI3K/Akt signaling. Int J Biochem Cell Biol 78: 75-86, 2016.

7. Doublier S, Lupia E, Catanuto P, Periera-Simon S, Xia X, Korach K, Berho M, Elliot SJ and Karl M: Testosterone and $17 \beta$-estradiol have opposite effects on podocyte apoptosis that precedes glomerulosclerosis in female estrogen receptor knockout mice. Kidney Int 79: 404-413, 2011.

8. Thomas PE, Wharram BL, Goyal M, Wiggins JE, Holzman LB and Wiggins RC: GLEPP1, a renal glomerular epithelial cell (podocyte) membrane protein-tyrosine phosphatase. Identification, molecular cloning, and characterization in rabbit. J Biol Chem 269: 19953-19962, 1994.

9. Aguiar RC, Yakushijin Y, Kharbanda S, Tiwari S, Freeman GJ and Shipp MA: PTPROt: An alternatively spliced and developmentally regulated B-lymphoid phosphatase that promotes G0/G1 arrest. Blood 94: 2403-2413, 1999. 
10. Alonso A, Sasin J, Bottini N, Friedberg I, Friedberg I, Osterman A Godzik A, Hunter T, Dixon J and Mustelin T: Protein tyrosine phosphatases in the human genome. Cell 117: 699-711, 2004.

11. Jacob ST and Motiwala T: Epigenetic regulation of protein tyrosine phosphatases: Potential molecular targets for cancer therapy. Cancer Gene Ther 12: 665-672, 2005.

12. Ramaswamy B, Majumder S, Roy S, Ghoshal K, Kutay H, Datta J, Younes M, Shapiro CL, Motiwala T and Jacob ST: Estrogen-mediated suppression of the gene encoding protein tyrosine phosphatase PTPRO in human breast cancer: Mechanism and role in tamoxifen sensitivity. Mol Endocrinol 23: 176-187, 2009.

13. Ozaltin F, Ibsirlioglu T, Taskiran EZ, Baydar DE, Kaymaz F, Buyukcelik M, Kilic BD, Balat A, Iatropoulos P, Asan E, et al: Disruption of PTPRO causes childhood-onset nephrotic syndrome. Am J Hum Genet 89: 139-147, 2011.

14. Charba DS, Wiggins RC, Goyal M, Wharram BL, Wiggins JE, McCarthy ET, Sharma R, Sharma M and Savin VJ: Antibodies to protein tyrosine phosphatase receptor type O (PTPro) increase glomerular albumin permeability [P(alb)]. Am J Physiol Renal Physiol 297: F138-F144, 2009.

15. Staněk L: Polymerase chain reaction: Basic principles and applications in molecular pathology. Cesk Patol 49: 119-121, 2013 (In Czech).

16. Hou J, Xu J, Jiang R, Wang Y, Chen C, Deng L, Huang X, Wang $X$ and Sun B: Estrogen-sensitive PTPRO expression represses hepatocellular carcinoma progression by control of STAT3. Hepatology 57: 678-688, 2013.

17. Rheault MN: Nephrotic and nephritic syndrome in the newborn. Clin Perinatol 41: 605-618, 2014

18. Zhao Y, Su B G, Xiao HJ, Zhang HW, Liu XY, Wang F and Ding J: Clinical characteristics of glucocorticoid-induced eye adverse reactions in children with primary nephrotic syndrome. Beijing Da Xue Xue Bao Yi Xue Ban 49: 794-797, 2017 (In Chinese).

19. Bettaieb A, Koike S, Hsu MF, Ito Y, Chahed S, Bachaalany S, Gruzdev A, Calvo-Rubio M, Lee KSS, Inceoglu B, et al: Soluble epoxide hydrolase in podocytes is a significant contributor to renal function under hyperglycemia. Biochim Biophys Acta 1861: 2758-2765, 2017.

20. Ito Y, Hsu MF, Bettaieb A, Koike S, Mello A, Calvo-Rubio M, Villalba JM and Haj FG: Protein tyrosine phosphatase 1B deficiency in podocytes mitigates hyperglycemia-induced renal injury. Metabolism 76: 56-69, 2017.

21. Kim NH: Podocyte hypertrophy in diabetic nephropathy. Nephrology (Carlton) 10 (Suppl): S14-S16, 2005

22. Delezay O, He Z, Hodin S, Saleem MA, Mismetti P, Perek N and Delavenne X: Glomerular filtration drug injury: In vitro evaluation of functional and morphological podocyte perturbations. Exp Cell Res 361: 300-307, 2017.

23. Abraham VC, Miller LN, Pratt SD, Putman B, Kim L, Gopalakrishnan SM and King A: Implementation of a human podocyte injury model of chronic kidney disease for profiling of renoprotective compounds. Eur J Pharmacol 815: 219-232, 2017.
24. Zhang S, Guo Y, Zou H, Sun N, Zhao D, Liu W, Dong Y, Cheng G and Yuan Q: Effect of estrogen deficiency on the fixation of titanium implants in chronic kidney disease mice. Osteoporos Int 26: 1073-1080, 2015.

25. Gluhovschi G, Gluhovschi A, Anastasiu D, Petrica L, Gluhovschi C and Velciov S: Chronic kidney disease and the involvement of estrogen hormones in its pathogenesis and progression. Rom J Intern Med 50: 135-144, 2012.

26. Ji H, Menini S, Mok K, Zheng W, Pesce C, Kim J, Mulroney S and Sandberg K: Gonadal steroid regulation of renal injury in renal wrap hypertension. Am J Physiol Renal Physiol 288: F513-F520, 2005.

27. Ming F and Sun Q: Epigenetically silenced PTPRO functions as a prognostic marker and tumor suppressor in human lung squamous cell carcinoma. Mol Med Rep 16: 746-754, 2017.

28. Wharram BL, Goyal M, Gillespie PJ, Wiggins JE, Kershaw DB, Holzman LB, Dysko RC, Saunders TL, Samuelson LC and Wiggins RC: Altered podocyte structure in GLEPP1 (Ptpro)-deficient mice associated with hypertension and low glomerular filtration rate. J Clin Invest 106: 1281-1290, 2000

29. Zou J, Yaoita E, Watanabe Y, Yoshida Y, Nameta M, Li H, Qu Z and Yamamoto T: Upregulation of nestin, vimentin, and desmin in rat podocytes in response to injury. Virchows Arch 448: 485-492, 2006

30. Barre B, Avril S and Coqueret O: Opposite regulation of myc and p21waf1 transcription by STAT3 proteins. J Biol Chem 278: 2990-2996, 2003.

31. Alvarez JV, Greulich H, Sellers WR, Meyerson M and Frank DA: Signal transducer and activator of transcription 3 is required for the oncogenic effects of non-small-cell lung cancer-associated mutations of the epidermal growth factor receptor. Cancer Res 66: 3162-3168, 2006.

32. Dudka AA, Sweet SM and Heath JK: Signal transducers and activators of transcription-3 binding to the fibroblast growth factor receptor is activated by receptor amplification. Cancer Res 70: 3391-3401, 2010.

33. Boccaccio C, Ando M, Tamagnone L, Bardelli A, Michieli P, Battistini $\mathrm{C}$ and Comoglio PM: Induction of epithelial tubules by growth factor HGF depends on the STAT pathway. Nature 391: 285-288, 1998

34. Song L, Turkson J, Karras JG, Jove R and Haura EB: Activation of Stat 3 by receptor tyrosine kinases and cytokines regulates survival in human non-small cell carcinoma cells. Oncogene 22: 4150-4165, 2003

35. Gu F, Dube N, Kim JW, Cheng A, Ibarra-Sanchez Mde J, Tremblay ML and Boisclair YR: Protein tyrosine phosphatase $1 \mathrm{~B}$ attenuates growth hormone-mediated JAK2-STAT signaling. Mol Cell Biol 23: 3753-3762, 2003.

36. Kleppe M, Soulier J, Asnafi V, Mentens N,Hornakova T, Knoops L, Constantinescu S, Sigaux F, Meijerink JP, Vandenberghe P, et al: PTPN2 negatively regulates oncogenic JAK1 in T-cell acute lymphoblastic leukemia. Blood 117: 7090-7098, 2011. 